\title{
Knowledge of intensive care nurses in selected care areas commonly guided by protocols
}

\author{
H Perrie, ${ }^{1}$ CCRN, MSc (Nursing); S Schmollgruber, ${ }^{1}$ CCRN, MSc (Nursing), PhD(c); J C Bruce, ${ }^{1}$ RN, MSc (Nursing), PhD; \\ P J Becker, ${ }^{1,2} \mathrm{MSc}, \mathrm{PhD}$ \\ 'Department of Nursing Education, Faculty of Health Sciences, University of the Witwatersrand, Johannesburg, South Africa \\ ${ }^{2}$ Biostatistics Unit, Medical Research Council, Pretoria, South Africa
}

Corresponding author: S Schmollgruber (shelley.schmollgruber@wits.ac.za)

\begin{abstract}
Objective. To assess the knowledge of nurses working in intensive care units (ICUs) in respect of pain management, glycaemic control and weaning from mechanical ventilation.

Methods. An analytical, cross-sectional survey design was used. All ICU-trained and non-ICU-trained registered nurses ( $N=136)$ working in the ICUs of selected public and private hospitals were invited to participate.

Results. The knowledge of both the ICU-trained and non-ICU-trained nurses was found to be lacking. The overall mean score (standard deviation) obtained was $47.56 \%$ (11.61). The ICU-trained participants obtained $50.11 \%$ (11.96) and non-ICU-trained participants obtained $45.01 \%$ (10.75). This difference, although small, was statistically significant $(p=0.0099)$. A poor relationship was found between level of knowledge and years of ICU experience.

Conclusion. Nurses lack knowledge in the three care areas tested. ICU nurses' experience cannot be relied upon as a source of knowledge for decision-making about protocol-directed care. It is suggested that on-going educational programmes be introduced into ICUs to address this shortfall in knowledge.
\end{abstract}

S Afr J Crit Care 2014;30(1):14-18. DOI:10.7196/SAJCC.158

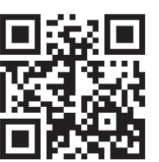

Critical care nurses have an important role to play in patient safety and ensuring that patients receive the best care possible. There has been a gradual shift away from basing nursing practice on tradition, expert opinion, trial and error and personal experience towards evidence-based practice (EBP). It is anticipated that in order to introduce EBP into intensive care units (ICUs), nursing care will increasingly be directed by guidelines and protocols. ${ }^{[1]} \mathrm{It}$ is argued that protocol-directed care can help to standardise the care received by patients regardless of user expertise. However, nursing care involves more than strictly applying the steps of a guideline or protocol, and includes having adequate knowledge to choose the best course of action for each patient care situation. Protocols and guidelines provide a framework from which nurses can work and are intended to facilitate or complement decision-making rather than replace it. ${ }^{[2,3]}$ Because of the multiple and complex critical illness conditions encountered in ICUs, it is very difficult for a single protocol to cover all the variables that may influence its application. Furthermore, a patient may be receiving care directed by more than one protocol, which may have steps that are incongruent with one another. ${ }^{[1]}$ This means that nurses must have and use specific knowledge competencies to guide their decisions when dealing with multiple protocols.

According to Toth, ${ }^{[4]}$ basic knowledge for ICU nurses is knowledge beyond that required to register as a nurse; it is this knowledge that nurses use to provide safe care to critically ill patients. Knowledge itself does not ensure safe practice but safe practice is not possible without knowledge. ${ }^{[4]}$ Since accountability forms the basis of professional nursing practice, it is up to each individual nurse to ensure that they have adequate knowledge in order to determine which aspects of a protocol are most suitable for the patient in any given situation. ${ }^{[5,6]}$ It is stated that safe practice is both a moral and professional responsibility of the nurse. Knowledge is therefore an important first step in providing safe patient care. Assessing the knowledge of ICU nurses allows for the introduction of suitable education programmes regarding protocols in ICUs. This may improve both patient safety and the individualised care of patients receiving protocol-directed care. This study assessed the knowledge of ICU-trained and non-ICU-trained nurses regarding pain management, glycaemic control and weaning from mechanical ventilation. These three care areas have been chosen as they are commonly directed by protocols.

\section{Objectives}

The objectives were to:

- describe the knowledge of nurses working in ICUs in respect of pain management, glycaemic control and weaning from mechanical ventilation

- compare the difference in knowledge between ICU-trained and non-ICU-trained nurses in respect of pain management, glycaemic control and weaning from mechanical ventilation.

\section{Methods}

An analytical, cross-sectional, survey design was used. This survey was conducted in three academic and two private sector hospitals in Johannesburg. A total of 13 ICUs were purposively selected for inclusion in the study. These ICUs were chosen to include nurses from both the public and private sectors to ensure as representative a sample as possible. All registered nurses working in the ICUs of these hospitals were invited to participate. 


\section{Ethical considerations}

Permission to conduct the study was obtained from the University's Postgraduate Committee and the Human Research Ethics Committee. Permission was also granted by the respective hospitals and the ICU nursing managers. Participation in the study was voluntary, and anonymity and confidentiality were assured. Signed informed consent was obtained from all participating nurses.

\section{Sample}

All registered nurses working in the selected ICUs and who were willing to participate and who had $>4$ months' experience in an ICU were included in the study. In consultation with the biostatistician it was calculated that a minimum sample size of 120 nurses $(N=120)$, i.e. 60 in each group (i.e. ICU trained and non-ICU trained), should be included in the study. Consecutive sampling was used and the sample size was achieved according to the response rate.

\section{Questionnaire}

Data were collected using a self-administered, multiple-choice questionnaire developed and validated following the method advocated by Lynn, ${ }^{[7]}$ using a two-stage process, namely the developmental and the quantification stages. The nursing experts used during this process were chosen for either their clinical or educational expertise in ICU. These experts were also asked to rate each item in the questionnaire as basic, applied or advanced according to the level of knowledge being tested. The definitions of level of knowledge were based on the three levels of competence (foundational, practical and reflective) as described by Morolong and Chabeli. ${ }^{[8]}$

After the validation process, the questions were arranged into a questionnaire consisting of three sections, namely pain management, glycaemic control and weaning from mechanical ventilation. Each section was introduced by a short case study followed by 11 multiple-choice questions, three of which were extended to assess the rationale/physiology behind the question. Demographic data, including age, nursing qualifications, employment sector and years of ICU experience, were obtained from all participants.

A pilot study was conducted with 10 nurses, 5 ICU-trained and 5 non-ICU-trained nurses from one of the academic hospitals included in the study. Following the pilot study, two of the questions were rephrased as they appeared too broad to elicit the expected answers. The results of the pilot study were not included in the final analysis. A competency score of $70 \%$ was set by expert ICU nurses as indicating an acceptable level of knowledge for nurses working in ICUs. It is generally accepted that the pass rate for a clinical assessment is $60 \%$ and for practical procedures $80 \%$. The competency indicator of $70 \%$ used in this study was therefore between the two levels. No distinction was made between the scores expected from the ICU-trained as opposed to the non-ICU-trained nurses as all nurses should be able to effectively manage the care of patients for whom they are responsible.

\section{Data collection}

Data were collected between February and March 2006. Nurses were asked to complete the questionnaire during on-duty time. The researchers remained in the units while the questionnaire was being completed to prevent data contamination. No emergencies occurred during the data collection periods, thereby allowing the nurses to complete the questionnaire while providing routine care to their patients. They placed the completed questionnaires in an envelope, which they sealed. Consent forms were placed in a separate envelope which was then sealed before being removed from each unit, thereby ensuring anonymity. The data were entered into a spread sheet designed with multiple data integrity checks.

\section{Data analysis}

Numbers and percentages were used to describe the demographics of the participants. As the data were normally distributed, means and standard deviations (SDs) were used to describe the level of knowledge. Comparison between the level of knowledge between ICU-trained and non-ICU-trained nurses was made using the Student's two-sample $t$-test. To ensure that years of experience did not influence the results, the groups were also compared with regard to mean assessment using an analysis of covariance (ANCOVA) with years of experience as a covariate. The impact of years of experience on the participants' level of knowledge was further assessed using Pearson's product moment correlation. Where appropriate, $95 \%$ confidence intervals (Cls) were reported. A significance level of 0.05 was used. Statistical analysis was performed using STATA 11 (2009) for Windows with the assistance of a biostatistician. Prior to analysis, $10 \%$ of the data were randomly checked for accuracy against the original surveys.

\section{Results}

Of the 142 questionnaires distributed, 136 were completed and returned (a $96 \%$ response rate).

\section{Demographics}

Fifty per cent $(n=68)$ of the nurses held an ICU qualification. Of the participants, $77.94 \%(n=106)$ were between the ages of 31 and 50 years with $14.71 \%(n=20)$ younger than 30 years and $7.35 \%(n=10)$ older than 50 years. The split between public and private sector nurses was $63.97 \%(n=87)$ and $36.03 \%(n=49)$ respectively. The mean ICU experience was 6.32 years for the total group, 8.45 years for the ICU-trained nurses and 4.14 years for the non-ICU-trained nurses. The demographic characteristics of participants are shown in Table 1 . This resulted in a statistically significant difference in the average years of experience between the two groups $(p=0.000)$

\section{Knowledge scores per section}

The mean score (SD) of all the participants for pain management was $43.97 \%(15.45 ; 95 \% \mathrm{Cl} 41.35$ - 46.59). The ICU-trained nurses achieved a mean score of $45.07 \%$ and the non-ICU-trained nurses $42.86 \%$. The mean difference was $2.2 \%$, which was not statistically significant $(p=0.4075)$.

For knowledge of glycaemic control, the mean score (SD) of all the participants was $48.71 \%(13.30 ; 95 \% \mathrm{Cl} 46.45$ - 50.97). The ICU-trained and the non-ICU-trained participants achieved mean scores of $51.26 \%$ and $46.16 \%$ respectively. The mean difference was $5.1 \%$, which was statistically significant $(p=0.0249)$.

The mean (SD) knowledge score of all the participants for weaning from mechanical ventilation was $50.00 \%$ (17.16; 95\% Cl 47.09 - 52.91). The mean score of the ICU-trained nurses was $53.99 \%$ and that of the non-ICU-trained nurses was $46.01 \%$. The mean difference was $7.98 \%$, which was statistically significant $(p=0.0063)$.

Overall the mean score (SD) of all the participants was $47.56 \%$ (11.61, (95\% Cl 45.59 - 49.52). ICU-trained nurses achieved a mean score of $50.11 \%(11.96,95 \% \mathrm{Cl} 47.21-53.00)$ and non-ICU-trained nurses $45.01 \%(10.75,95 \% \mathrm{Cl} 42.40-47.61)$. The mean difference between the two groups was $5.1 \%$. This difference was statistically significant ( $p=0.0099$ ). These results are shown in Table 2 . 


\section{Knowledge scores per level of knowledge}

Knowledge items in the questionnaire were categorised as basic, applied and advanced. Nurses' mean score (SD) for the 13 basic knowledge questions was $55.14 \%$ (16.90, $95 \%$ Cl 52.27 - 58.00). ICU-trained nurses achieved a mean score of $58.58 \%$ (15.82, $95 \% \mathrm{Cl} 54.75$ - 62.41) whereas the non-ICUtrained nurses achieved a mean score of $51.69 \%$ (17.35, 95\% Cl 47.49 - 55.89). The difference in basic knowledge was $6.89 \%$, which was statistically significant $(p=0.0169$ ).

The mean scores for the 17 applied knowledge questions were similar to the basic knowledge questions and were the same for both groups. The mean score for the sample $(N=136)$ was $56.66 \%(13.22 ; 95 \% \mathrm{Cl} 54.42$ 58.90). The mean score of ICU-trained nurses was $56.66 \%(13.93,95 \% \mathrm{Cl} 53.29$ - 60.03) and that of the non-ICU-trained nurses was also $56.66 \%$ (12.57, 95\% Cl 53.61 - 59.70). Statistically there was no difference between the two groups ( $p=0.9990)$.

Nurses' mean score (SD) for the 12 advanced knowledge questions was $26.44 \%$ (15.92, $95 \% \mathrm{Cl} 23.74$ - 29.14). ICU-trained nurses achieved a mean score of $31.61 \%(16.83$, 95\% Cl 27.54 - 35.69) and the non-ICU-trained participants $21.26 \%(13.16,97 \%$ Cl 18.08 24.45). The mean difference between the two groups was $10.35 \%$, resulting in a statistically significant difference $(p=0.0001)$ (Table 3$)$.

\section{Knowledge correlated with years of experience}

When the extent to which knowledge of nurses was influenced by years of ICU experience was tested, a correlation coefficient of 0.137 was found; this indicates a weak positive relationship between nurses' knowledge and their experience in ICU, which was not statistically significant $(p=0.1142)$.

\section{Discussion}

The results support several other studies that have shown knowledge deficits in different aspects of nursing care. The average knowledge score for ICU nurses was $47.56 \%$, with only four participants achieving a mark at or above $70 \%$. This lack of knowledge may make it difficult for the nurses to comply with, or make informed decisions when implementing protocol-directed care, particularly if steps in different protocols are incongruent with one another.

Scores of $55.14 \%$ and $56.66 \%$, respectively, were achieved for the items addressing basic and applied knowledge which, although low,
Table 1. Demographics of ICU nurses ( $N=136)$

\begin{tabular}{|c|c|c|c|}
\hline Characteristics & $\begin{array}{l}\text { Total sample of } \\
\text { ICU nurses } \\
(N=136)\end{array}$ & $\begin{array}{l}\text { ICU-trained } \\
\text { nurses } \\
(n=68)\end{array}$ & $\begin{array}{l}\text { Non-ICU-trained } \\
\text { nurses } \\
(n=68)\end{array}$ \\
\hline \multicolumn{4}{|l|}{ Age, years, $n(\%)$} \\
\hline $20-30$ & $20(14.71)$ & $7(10.29)$ & $13(19.12)$ \\
\hline $31-40$ & $60(44.12)$ & $24(35.29)$ & $36(52.94)$ \\
\hline $41-50$ & $46(33.82)$ & 31 (45.59) & $15(22.06)$ \\
\hline $51-60$ & $10(7.35)$ & $6(8.82)$ & $4(5.88)$ \\
\hline \multicolumn{4}{|c|}{ Employment sector, $n$ (\%) } \\
\hline Public & $87(63.97)$ & $45(66.18)$ & $42(61.76)$ \\
\hline Private & $49(36.03)$ & $23(33.82)$ & $26(38.42)$ \\
\hline \multicolumn{4}{|c|}{ ICU experience, years } \\
\hline Range & $0.3-25$ & $1-25$ & $0.3-25$ \\
\hline Mean & 6.32 & 8.45 & 4.14 \\
\hline SD & 5.21 & 5.19 & 4.23 \\
\hline
\end{tabular}

Table 2. Results of ICU nurses' knowledge per care area

\begin{tabular}{|c|c|c|c|c|c|}
\hline & $\begin{array}{l}\text { Mean } \\
\text { score } \\
(\%)\end{array}$ & SD & $95 \% \mathrm{Cl}(\%)$ & $\begin{array}{l}\text { Difference } \\
\text { between } \\
\text { groups (\%) }\end{array}$ & $p$-value \\
\hline \multicolumn{6}{|l|}{ Pain management } \\
\hline ICU-trained & 45.07 & 16.01 & $41.19-48.94$ & & \\
\hline Non-ICU-trained & 42.86 & 14.91 & $39.26-46.47$ & 2.20 & 0.4075 \\
\hline \multicolumn{6}{|l|}{ Glycaemic control } \\
\hline ICU-trained & 51.26 & 11.74 & $48.42-54.10$ & & \\
\hline Non-ICU-trained & 46.16 & 14.34 & $42.69-49.63$ & 5.10 & 0.0249 \\
\hline \multicolumn{6}{|c|}{$\begin{array}{l}\text { Weaning from mechanical } \\
\text { ventilation }\end{array}$} \\
\hline ICU-trained & 53.99 & 18.19 & $49.59-58.40$ & & \\
\hline Non-ICU-trained & 46.01 & 15.18 & $42.34-49.68$ & 7.98 & 0.0063 \\
\hline \multicolumn{6}{|c|}{$\begin{array}{l}\text { Composite score for protocol } \\
\text { directed care areas }\end{array}$} \\
\hline ICU-trained & 50.11 & 11.96 & $47.21-53.00$ & & \\
\hline Non-ICU-trained & 45.01 & 10.75 & $42.40-47.61$ & 5.10 & 0.0099 \\
\hline
\end{tabular}

Table 3. Results for level of knowledge of ICU nurses

\begin{tabular}{|c|c|c|c|c|c|}
\hline & $\begin{array}{l}\text { Mean } \\
\text { score } \\
(\%)\end{array}$ & SD & $95 \% \mathrm{Cl}$ & $\begin{array}{l}\text { Difference } \\
\text { between } \\
\text { groups (\%) }\end{array}$ & $p$-value \\
\hline \multicolumn{6}{|l|}{ Basic level } \\
\hline ICU-trained & 58.58 & 15.82 & $54.75-62.41$ & & \\
\hline Non-ICU-trained & 51.69 & 17.35 & $47.49-55.89$ & 6.89 & 0.0169 \\
\hline \multicolumn{6}{|l|}{ Applied level } \\
\hline ICU-trained & 56.66 & 13.93 & $53.29-60.03$ & & \\
\hline Non-ICU-trained & 56.66 & 12.57 & $53.61-59.70$ & 0.00 & 0.9990 \\
\hline \multicolumn{6}{|l|}{ Advanced level } \\
\hline ICU-trained & 31.61 & 16.83 & $27.54-35.69$ & & \\
\hline Non-ICU-trained & 21.26 & 13.16 & $18.08-24.45$ & 10.35 & 0.0001 \\
\hline
\end{tabular}


may indicate that the participants in this study are to some extent able to rationalise their actions and decisions. Advanced knowledge levels were very low with an average score of $26.44 \%$, indicating that the clinical rationale behind many actions and decisions may be lacking.

Several studies have shown knowledge deficits among nurses regarding routine ICU practices. In a local study by Windsor, ${ }^{[0]}$ on the interpretation of ventilator graphics by ICU nurses, a mean score of $40.3 \%$ was obtained. Two studies, which included 22 European countries, found that ICU nurses lacked knowledge of guidelines for the prevention of ventilator-associated pneumonia ${ }^{[10]}$ and the prevention of central venous catheter-related infections. ${ }^{[1]}$

Knowledge of pain management was poor (43.97\%). Although pain is associated with many complications, it is possible that in a busy, stressful ICU environment it may be given lower priority than other aspects of care. This finding is consistent with other studies assessing nurses' knowledge of pain management. ${ }^{[12,13]}$ Nurses' poor knowledge scores for glycaemic control (48.71\%) were unexpected because since publication of the Van den Berghe et al. study, ${ }_{1}^{[14]}$ glycaemic control has become part of everyday discussion and practice in ICUs. Furthermore, knowledge of normal blood glucose values, insulin administration and the signs and symptoms of hypoglycaemia are not specific to ICUs but are required in all fields of nursing.

Although nurses scored highest on weaning patients from mechanical ventilation, the mean score of $50 \%$ is below what is expected from ICU nurses. The slightly higher score may be explained by the fact that the most common reason for admission of an adult patient to an ICU is the need for mechanical ventilation ${ }^{[15]}$ and that approximately $41 \%$ of time required for ventilation is spent on the weaning process. ${ }^{[16]} \mathrm{ICU}$ nurses therefore may have had more exposure to this aspect of care than other care areas addressed in this study.

There was a distinct knowledge deficit with regard to the advanced knowledge items, which required substantiation or explanation of a response to the preceding question. This indicates that many actions by ICU nurses in respect of pain management, glycaemic control and weaning from mechanical ventilation are being carried out without knowledge of the physiological rationale behind the action.

Apart from knowledge levels, factors such as years of experience and level of qualification have been found to influence nurses' decision-making in ICUs. ${ }^{[17]}$ The difference of $5.1 \%$ between the ICUqualified nurses, who have completed specialist education in ICU nursing and non-ICU-qualified nurses in this study was small but statistically significant $(p=0.0099)$. The knowledge studies by Labeau et al. ${ }^{[10,11]}$ also found a small, but not statistically different difference between the scores of nurses holding an ICU qualification and those without. Both groups' low scores and the small difference between their scores is of concern as ICU-qualified nurses are expected to have an advanced knowledge base in order to make sound clinical judgements within the ICU environment, and should function at a higher level than those nurses without the specialty qualification. ${ }^{[6]}$

In this study, years of ICU experience did not influence knowledge levels; only a weak correlation ( $r=0.137$ ) was found between knowledge levels and years of experience. This finding is in keeping with another local study where the most highly qualified ICU nurses (those with a Master's degree) achieved the lowest scores. ${ }^{[9]}$ Toth $^{[4]}$ however found when comparing basic knowledge in ICUs between nurses from the USA and other countries that ICU nurses with more years of experience consistently achieved higher scores on knowledge tests.

Ensuring that critically ill patients receive safe, high-quality care in a complex ICU environment is an ongoing challenge. This study reported on the knowledge of ICU nurses and may therefore not reflect the actual practice environment; however, it can be inferred to some extent that the results reflect practice. ${ }^{[1]}$ There is an assumption that knowledge improves decision-making and therefore patient outcomes, but whether knowledge translates to better bedside care is not known. ${ }^{[18]}$ Toth $^{[19]}$ states that, 'although basic knowledge does not guarantee safe practice, safe practice in critical care nursing cannot occur without basic knowledge'. Giuliano and Liu ${ }^{[20]}$ confirm this by stating that it is not possible to provide appropriate care to patients without adequate knowledge of the specific area of care.

\section{Study limitations}

The limitations of this study include the relatively small sample size and the limited geographical area in which it was conducted, this being constrained by the time and resources available. Consecutive sampling does not allow generalisation of our results to all nurses working in ICU. Not having an 'I do not know' option may have encouraged participants to guess when they were not sure of the answers. This study only evaluated knowledge regarding specific care areas commonly directed by protocols and not actual practice or adherence to protocols.

\section{Recommendations}

Educational programmes need to be introduced into ICUs to improve knowledge regarding pain management, glycaemic control and weaning from mechanical ventilation. As the staff turnover in many ICUs is high, the programmes need to be repeated regularly. In order to ensure that patients in ICUs receive care from knowledgeable, competent nursing staff a continuing professional development system should become compulsory for nurses. Further research could address the impact of protocols on both nurses'knowledge and on patient outcome.

\section{Conclusion}

This study identified gaps in ICU nurses' knowledge regarding three care areas commonly guided by protocols. The participants had knowledge levels below the required competency score of $70 \%$. This applies to all levels of knowledge: basic, applied and advanced, and to all three areas of protocol-directed care. Years of experience are poorly correlated to ICU nurses' knowledge and cannot be relied upon as a source of knowledge for decision-making about protocol-directed care. Providing evidence-based care has become important in ICU nursing, along with nurses increasingly having to take responsibility for their actions. Without appropriate knowledge, complications are more likely to occur, even when delivering protocol-directed care.

Acknowledgements. We like to acknowledge the contribution of the nurses, without whom this study would not have been possible.

\section{References}

1. Hewitt-Taylor J. Clinical guidelines and care protocols. Intensive Crit Care Nurs 2004;20(1):45-52 [http://dx.doi.org/10.1016/j.iccn.2003.08.002]

2. Considine J, Hood K. Emergency Department management of hip fractures: Development of an evidence-based clinical guideline by literature review and consensus. Emerg Med 2000;12(4):329-336. [http://dx.doi.org/10.1046/j.1442-2026.2000.00157.x]

3. Ely EW, Meade MO, Haponik EF, et al. Mechanical ventilator weaning protocols driven by nonphysician health-care professionals: Evidence-based clinical practice guidelines. Chest 2001;120(6 Suppl):454S-4635.

4. Toth JC. Comparing basic knowledge in critical care nursing between nurses from the United States and nurses from other countries. Am J Crit Care 2003;12(1):41-46.

5. Thomson P, Angus NJ, Scott J. Building a framework for getting evidence into critical care education and practice. Intensive Crit Care Nurs 2000;16(3):164-174. [http://dx.doi.org/10.1054/iccn.2000.1483]

6. Searle C. Professional Practice: A Southern African Nursing Perspective. 3rd ed. Johannesburg: Heinemann, 2002.

7. Lynn MR. Determination and quantification of content validity. Nurs Res 1986;35(6):382-385.

8. Morolong BG, Chabeli MM. Competence of newly qualified registered nurses from a nursing college. Curationis 2005;28(2):38-50.

9. Windsor S. An educational programme for critical care nurses on the interpretation of ventilator graphics (unpublished dissertation, MSc Nursing). University of Pretoria, 2005. 
10. Labeau S, Vandijck D, Rello J, et al. Evidence-based guidelines for the prevention of ventilatorassociated pneumia Results of a knowledge test among European intensive care nurses. Hosp Infect 2008;70(1):180-185. [http://dx.doi.org/ 10.1016/j.jhin.2008.06.027]

11. Labeau SO,VandijckDM, Rello J, et al.Centers for Disease Control and Prevention guidelines for preventing central venous catheter-related infection: Results of a knowledge test among 3405 European intensive care nurses. Crit Care Med 2009;37(1):320-323. [http://dx.doi.org/10.1097/CCM.0b013e3181926489]

12. Fothergill-Bourbonnais $F$, Wilson-Barnett $J$. A comparative study of intensive therapy unit and hospice nurses' knowledge on pain management. J Adv Nurs 1992;17(3):362-372.

13. Erkes EB, Parker VG, Carr RL, et al. An examination of critical care nurses'knowledge and attitudes regarding pain management in hospitalized patients. Pain Manag Nurs 2001;2(2):47-53. [http:// dx.doi.org/10.1053/jpmn.2001.23177]

14. Van den Berghe $G$, Wouters $P$, Weekers $F$, et al. Intensive insulin therapy in critically ill patients. N Engl J Med 2001;345(19):1359-1367. [http://dx.doi.org/10.1056/NEJMoa011300]
15. Tobin MJ. Advances in mechanical ventilation. N Engl J Med 2001;344(26):1986-1996. [http:// dx.doi.org/10.1056/NEJM200106283442606]

16. Estban A, Alia I, Ibanez J, et al. Modes of mechanical ventilation and weaning: A national survey of Spanish hospitals. Chest 1994;106(4):1188-1194.

17. Pirret AM. The level of knowledge of respiratory physiology articulated by intensive care nurses to provide rationale for their clinical decision-making. Intensive and Critical Care Nursing 2007;23(3):145-155. [http://dx.doi.org/10.1016/j.iccn.2006.11.004]

18. Kendall-Gallagher D, Blegen MA. Competence and certification of registered nurses and safety of patients in intensive care units. Am J Crit Care 2009;18(2):106-116. [http://dx.doi.org/10.4037/ajcc2009487]

19. Toth JC. Evaluating the use of the Basic Knowledge Assessment Tool (BCAT) in critical care nursing with baccalaureate nursing students. Image J Nurs Sch 1984;16(3):67-71.

20. Giuliano KK, Liu LM. Knowledge of pulse oximetry among critical care nurses. Dimens Crit Care Nurs 2006;25(1):44-49. 